\title{
Effectiveness of 2 Methods of Promoting Physical Activity, Healthy Eating, and Emo- tional Well-Being With the Americans in Motion-Healthy Interventions Approach
}

\author{
Wilson D. Pace, $M D^{1}$ \\ Angela M. Lanigan, MPA, RD ${ }^{2}$ \\ Elizabeth W. Staton, MSTC ${ }^{1}$ \\ Deborab G. Grabam, MSPH \\ Brian K. Manning, MPH \\ L. Miriam Dickinson, $P b D^{1}$ \\ Caroline B. Emsermann, $M S^{1}$ \\ Elizabeth E. Stewart, $P b D^{1}$ \\ 'American Academy of Family Physicians \\ National Research Network, Leawood, \\ Kansas
}

${ }^{2}$ American Academy of Family Physicians National Research Network, Leawood, Kansas?

${ }^{3}$ Saint Luke's Health System, Kansas City, Missouri

\begin{abstract}
PURPOSE An increasing number of Americans are putting their health at risk from being overweight. We undertook a study to compare patient-level outcomes of 2 methods of implementing the Americans In Motion-Healthy Interventions (AIM-HI) approach to promoting physical activity, healthy eating, and emotional well-being.
\end{abstract}

METHODS We conducted a randomized trial in which 24 family medicine practices were randomized to (1) an enhanced practice approach in which clinicians and office staff used AIM-HI tools to make personal changes and created a healthy environment, or (2) a traditional practice approach in which physicians and staff were trained and asked to use the tools with patients. Of the 610 patients enrolled, 331 were in healthy practices, and 279 were in traditional practices. At 0, 4, and 10 months we assessed blood pressure, body mass index, fasting blood glucose and insulin levels, nuclear magnetic resonance lipoprotein profiles, fitness, dietary intake, physical activity, and emotional well-being. Outcome data were analyzed using linear, mixed-effects multivariate models, adjusting for practices as a random effect.

RESULTS Regardless of patient group, $16.2 \%$ of patients who completed a 10 -month visit ( $n=378$ patients, $62 \%$ of enrollees) and $10 \%$ of all patients enrolled lost $5 \%$ or more of their body weight; $16.7 \%$ of patients who completed a 10 -month visit (10.3\% of all enrollees) had a 2 -point or greater increase in their fitness level; and $29.2 \%$ of 10 -month completers (18.0\% of all enrollees) lost $5 \%$ or more of their body weight and/or increased their fitness level by 2 or more points. There were no significant differences in these outcomes between groups.

CONCLUSIONS There was no difference between the 2 groups in the primary and most secondary outcomes. Both patient groups were able to show significant before-after improvements in selected patient-level outcomes.

Ann Fam Med 2013;371-380. doi:10.1370/afm.1516.

\section{INTRODUCTION}

Conflicts of interest: authors report none.

\section{CORRESPONDING AUTHOR}

Wilson D. Pace, MD

Department of Family Medicine

University of Colorado

Mail Stop F496

12631 E 17th Ave

Aurora, CO 80045

Wilson.pace@ucdenver.edu
7 o effectively manage the increasing number of patients with obesity-related chronic illnesses, the medical community should embrace a major role in encouraging patients to make healthy lifestyle choices. Primary care is well suited to fill this need, because most of the US population identifies a primary care clinician as their usual source of care. ${ }^{1}$ Americans In Motion-Healthy Interventions (AIM-HI) is a public health initiative of the American Academy of Family Physicians. ${ }^{2}$ The initiative promotes healthy lifestyle choices related to nutrition, physical activity and emotional well-being. AIM-HI provides family physicians with tools to help promote attention to fitness-which is interpreted as 
adequate physical activity, healthy eating, and emotional well-being - as the cornerstone of health for all individuals. ${ }^{3}$ Although not specifically focused on obesity, the AIM-HI approach can be used to help individuals for whom surgery is not the treatment of choice (body mass index of greater than 40).

The AIM-HI program was developed around the transtheoretical model of behavioral change, ${ }^{4}$ which begins with identifying patients' readiness for lifestyle change. The interventional approach is based on changes within a person's current daily activities. ${ }^{5} \mathrm{Cli}$ nicians engage patients in a nonprescriptive approach by means of motivational interviewing ${ }^{6}$ to help patients develop goals that are feasible and personalized. AIM$\mathrm{HI}$ is based on a small-changes method of behavior change $\mathrm{e}^{7-9}$ with a focus on intuitive eating ${ }^{10}$ and attention to the interaction between emotional well-being and successful behavioral change. ${ }^{11}$ The full initiative calls for a 3-step process: clinicians are asked to evaluate their personal behaviors, the practice is asked to create a healthy environment and demonstrate this environment to patients, and the practice then engages individual patients in improving their health behaviors The 3 -step process is based on literature that indicates physicians who engage in healthy behaviors are more likely to counsel their patients in these areas. ${ }^{12-14}$

We studied the results, at the practice and the patient level, of implementing the AIM-HI program. In this report, we compare patient-level outcomes between practices randomized to 2 different methods of implementing the AIM-HI tools. Outcomes of the patientlevel randomization will be reported in a separate article.

\section{METHODS}

\section{Research Design}

This study evaluated the effectiveness of the AIM$\mathrm{HI}$ educational tools and motivational interviewing approaches on individual patient behavior change. Behavior change was determined by change in physiologic measures and patient self-reported diet, physical activity, and emotional well-being. The study included a $2 \times 2$ factorial design with randomization at the practice and patient levels.

Practices were randomized to an enhanced practice approach that involved both clinicians and office staff making personal changes and creating a healthy practice environment. The enhanced practices were compared with traditional practices, which were trained and asked to use the tools directly with patients. In this article, we report on the practice randomization aspect of the study.

Patients in both traditional and enhanced practices were randomized to either receive enhanced feedback or limited feedback. Patients receiving limited feedback and their clinicians were given patients' weight and self-reported physical activity and quality of life scores. Patients receiving enhanced feedback and their clinicians were given the same, plus the results of 2 physiologic measures that may change more quickly than classic biomarkers, such as weight, blood pressure, and total cholesterol or low-density lipoprotein (LDL) cholesterol, with improvements in physical activity and diet: homeostatic model assessment-insulin resistance (HOMA-IR) and nuclear magnetic resonance lipoprotein profiles (NMRLP). Insulin resistance typically increases with increasing body mass index (BMI) and is considered a precursor to type 2 diabetes mellitus. ${ }^{15}$ The absolute number of LDL cholesterol particle numbers, as measured by NMRLP, have been correlated with cardiovascular disease risk ${ }^{16,17}$ and have been shown to be responsive to changes in physical activity $^{18}$ and weight. ${ }^{19}$

\section{Setting and Participants}

Twenty-four family medicine practices in 16 US states were selected from 63 practices that originally responded to the study recruitment notices from 3 separate practice-based research networks: the American Academy of Family Physicians National Research Network, LA Net, and Southeast Clinicians Research Network. Forty-seven practices were assessed for eligibility, and the final 24 were selected based on their current level of activity related to lifestyle interventions, underlying socioeconomic make-up of the practice population, racial and ethnic diversity (with an expressed intent to recruit 12 practices with greater that $40 \%$ of the patient population consisting of individuals of African American, Native American/Alaska Native or Hispanic descent), and geographic diversity. During the course of the study, 3 practices dropped out because of organizational changes.

The AIM-HI intervention was designed to be provided to all appropriate patients in a practice, but it was evaluated among a sample of patients from each practice. Each practice enrolled up to 40 patients who were randomly assigned to either the enhanced feedback or limited feedback groups. Patients were eligible if they were older than 17 years, had a BMI of 30 or greater, were able to participate in moderate physical activity, had a life expectancy greater than 1 year, and were able to read English or Spanish. Exclusion criteria included a diagnosis of coronary heart disease, human immunodeficiency virus infection/acquired immunodeficiency syndrome, or hepatitis C. Pregnant women and those planning to become pregnant in the next 12 months were also excluded. Initially, patients with a diagnosis of type 2 diabetes or hyperlipidemia before the index 
visit were not eligible for the study. This criterion was relaxed approximately 3 months after the initiation of patient enrollment as long as the condition and treatment regimen had been stable for at least 6 months.

\section{Fitness Intervention}

The AIM-HI approach focuses on facilitating a conversation between clinicians and their patients via motivational interviewing ${ }^{20}$ with the purpose of supporting patients' attempts to make incremental behavior changes. The physician or other member of the practice team and patient together decided which AIM-HI tools were most appropriate for the patient's needs and set goals accordingly. The AIM-HI tools included a fitness inventory, fitness prescription, and food and activity journal. ${ }^{3}$ Practices developed approaches to integrate the AIM-HI tools into routine clinical care. Most practices focused on using the tools during prevention and wellness visits. The recommended approach was to screen individuals with the fitness inventory. This tool assesses current behaviors related to the 3 domains and readiness to change. This instrument was then used by the clinician to explore whether a patient was interested in changing behaviors in 1 of 3 domains. Patients were encouraged to develop their own approaches to improvements by making small, incremental changes. For healthy eating, a nondiet approach was encouraged. ${ }^{10}$ The clinician then wrote the planned changes and a follow-up plan on a fitness prescription that was signed by the clinician and patient. Patients could use the food and activity journal to document their feeling and emotions during recorded activities. The AIM-HI Toolkit is available from the American Academy of Family Physicians. ${ }^{3}$

\section{Test and Control Conditions}

Twenty-four practices were randomized into either the enhanced practice group or the traditional practice group. Practices randomized into the enhanced practice group were introduced to the AIM-HI tools 4 months before they were to begin using the materials with patients. One clinician and 1 staff member from each practice participated in a 1.5-day training session during which the AIM-HI philosophy and tools were introduced and the participants practiced using them. These individuals were then asked to champion the use of these tools and consider other practice-level activities to promote clinician and office staff improvements in their own behaviors. All staff and clinicians in enhanced practices were encouraged to become familiar with the tools, use the tools to make their own personal lifestyle changes, and create a fitness culture (ie, healthy practice) within their own practices. Practices in the traditional group were trained in the use of the AIM-HI tools at a second 1.5-day session that occurred approximately 3 months after the enhanced practice training. The 2 sessions were similar, but the traditional practices were encouraged to use the AIMHI tools with patients but not instructed to initiate practice-level interventions.

Patients in both the enhanced practice and the traditional practice groups were randomized into the enhanced or limited feedback groups. Limited feedback patients made 3 office visits with the practice study coordinator for data collection and feedback: baseline, 4 months, and 10 months. Enhanced feedback patients made these same visits plus 2 additional visits at 2 months and 7 months. Both groups of patients could make other office visits focused on their behavioral change activities as agreed upon by the patient and clinician.

Clinical data collected during study visits included blood pressure, weight, height, fasting glucose and insulin levels, and NMRLP. ${ }^{19,21}$ BMI and HOMA-IR were calculated from these data. HOMA-IR is an estimate of insulin resistance, and NMRLP measures multiple components of cholesterol transport within the blood. Both measures appear to change with changes in weight or physical fitness levels. These tests have not been widely introduced into primary care clinical practice.

At baseline, 4-, and 10-month visits, all study patients also completed a 3 -minute step test and the following 5 self-report instruments: the PrimeScreen (a brief dietary screening tool), ${ }^{22}$ International Physical Activity Questionnaire, ${ }^{23}$ Treatment Self-Regulation Questionnaire, ${ }^{24}$ the visual analog scale from the EQ-5D health state score, ${ }^{25,26}$ and the SF-12. ${ }^{27}$ These validated instruments provide data on physical activity, motivation, eating habits, and emotion well-being. The 3 -minute step test was conducted on an 8 -inch step using a metronome at a rate of 96 steps per minute. Heart rate was measured immediately after completing 3 minutes of stepping and again 1 minute later. The pulse obtained at the end of the 3 -minute step test was converted to a fitness level based on age and sex according to published standards. Fitness scores range from 1 to 7, with higher scores indicating greater levels of physical fitness. The International Physical Activity Questionnaire was square root transformed to normalize the results for analysis. ${ }^{28}$

All instruments were scored according to published standards. A secondary PrimeScreen score (PrimeScreen AIM-HI) was also developed to track more appropriately with the AIM-HI intuitive eating concepts and was used for all analyses in this article (this scoring approach is available upon request). The main study outcome was a combined measure of change in BMI, change in fitness level, and change in SF-12 men- 
tal health scores (Combined Outcome Score). These 3 metrics address each area within the AIM-HI toolkit with either physiologic measures or a well-validated assessment of mental well-being.

The project sought to have 204 patients complete the intervention per study arm. Because of a combination of practices leaving the study and patients not completing the study, this number was not achieved. Patients completing the study at 10 months allowed the project to detect a 0.43 effect size with the observed intraclass correlation coefficient of 0.04 , which equates to an 11-point difference in the combined outcome measure. The study was not powered on analyses of individual measures.

Study staff visited all practices twice during the study and frequently (typically weekly) engaged designated practice members by telephone or e-mail contact. An experienced qualitative analyst (E.E.S.), blinded to the original data, reviewed all site visit and contact notes and developed an independent scoring system to assess the degree of intervention fidelity. Data were organized into 2 metrics: metric 1 examined the degree to which practices used AIM-HI tools and strategies as part of patient care (eg, fitness inventory); metric 2 examined the degree to which practices created an enhanced practice environment (eg, pedometer contests, healthy potlucks) regardless of practice assignment. Metric 2, practice wellness, included 6 domains scored from 1 to 3 (for a scale of 6 to 18). To further validate the scores, all individual subscores underwent a second level of review, nonblinded, by study staff who collected the data. Each practice received 2 composite scores (tools and strategies, practice wellness) to represent intervention fidelity. The practice wellness score was included in this analysis, which focuses on practice-level randomization.

\section{Data Analysis}

Linear mixed effects multivariate models, adjusting for practices as a random effect, were performed for the outcome measures BMI, systolic blood pressure, fitness score (based on the 3-minute step test), PrimeScreen AIM-HI score, SF-12 mental component summary score, and EQ-5D health state score. Each model adjusted for survey (baseline, 4 months, 10 months), a practice measure presented below, and survey by practice measure interaction. Before the analyses, mixed models adjusting only for survey determined a nonlinear relationship between time and the outcomes; therefore, time was run as a categorical predictor.

\section{Practice Measures}

Two analyses were performed that adjusted for practice based on (1) the randomized practice group (enhanced vs traditional) and (2) a composite score (scale 6 to 18) representing practice wellness

For analysis 1 all patients and practices were included. For analysis 2, practices 36 and 37 were combined. In these practices, clinicians from 1 practice covered the other practice for extended periods and therefore were no longer independent entities. In addition, 3 practices $(17,38,39)$-accounting for 13 respondents-dropped out of the study and therefore did not have wellness or tool composite scores. These practices were not included in these analyses. Composite measures were standardized before the analysis. The reasons for dropout were 2 practices were bought by corporate entities and could not continue the project during the transition, and in 1 practice the only physician left.

\section{Models}

All models adjusted for patient characteristics that include age centered at the mean, number of comorbidities, education level, sex, income, marital status, and race, and ethnicity. In addition, 1 degree-of-freedom hypotheses were made to determine differences between baseline to 4 -month and 10-month averages. For analysis 2, comparisons were made for practices 1 standard deviation below the mean composite score, at the mean composite score, and 1 standard deviation above the mean composite score. Statistically significant associations were determined at the $\alpha=.05$ level. All analyses were performed in SAS 9.2 (SAS Institute).

The protocol for this study was approved by the American Academy of Family Physicians Institutional Review Board and the institutional review boards covering participating practices.

\section{RESULTS}

Table 1 displays demographic information for the traditional and enhanced practice patient cohorts. There were only 2 statistically significant differences between the 2 groups: marital status and number of people living in the home. Table 2 displays the baseline outcome measures of the traditional and enhanced practice patient cohorts. Again, there are few differences between the 2 groups, with blood pressure and NMRLP being lower in the traditional practice group. These data indicate that the practice-level, block randomization scheme resulted in reasonably similar patient-level cohorts as measured by these variables. The data also indicate that the expressed intent of the study, to oversample minority populations, was achieved with more than $40 \%$ of the population being non-White and $16 \%$ being of Latino/Hispanic ethnicity. These data also indicate that the intent of the 
study to enroll individuals across the income spectrum was achieved. Figures 1 and 2 show the CONSORT diagrams for practices and patients.

Table 3 displays the baseline and 4 - and 10 -month primary and secondary clinical outcomes divided by practice-level randomization. At baseline the traditional practice cohort had significantly lower systolic and diastolic blood pressure, but the groups were otherwise reasonably well matched. There was only 1 significant difference in these outcomes between the enhanced and traditional practices with time: lowering of systolic blood pressure in the enhanced practices. Both groups showed greater changes at 4 months than 10 months, though statistically significant improvements were seen in multiple measures at 10 months

\section{Table 1. Demographic Characteristics of Patients in Enhanced and Traditional Practices}

\begin{tabular}{|c|c|c|c|}
\hline Variable & $\begin{array}{c}\text { Enhanced } \\
\text { Practice } \\
n=331 \\
\text { No. }(\%)\end{array}$ & $\begin{array}{c}\text { Traditional } \\
\text { Practice } \\
n=279 \\
\text { No. }(\%)\end{array}$ & $\begin{array}{c}P \\
\text { Value }^{a}\end{array}$ \\
\hline Sex, female & $255(77.0)$ & $211(75.6)$ & .683 \\
\hline \multicolumn{4}{|l|}{ Racial background } \\
\hline White & $186(56.2)$ & $165(59.1)$ & .828 \\
\hline African-American & $57(17.2)$ & $46(16.5)$ & \\
\hline Other & $62(18.7)$ & $45(16.1)$ & \\
\hline Missing & $26(7.9)$ & $23(8.2)$ & \\
\hline \multicolumn{4}{|l|}{ Ethnicity } \\
\hline Not Hispanic/Latino & $272(82.2)$ & $240(86.0)$ & .198 \\
\hline Hispanic/Latino & $59(17.8)$ & $39(14.0)$ & \\
\hline \multicolumn{4}{|l|}{ Education } \\
\hline Less than high school & $19(5.7)$ & $8(2.9)$ & .330 \\
\hline High school grad/GED & $85(25.7)$ & $74(26.5)$ & \\
\hline Some college, graduate school & $202(61.0)$ & $171(61.3)$ & \\
\hline Missing & $25(7.6)$ & $26(9.3)$ & \\
\hline \multicolumn{4}{|l|}{ Income } \\
\hline$<\$ 20,000$ & $70(21.1)$ & $55(19.7)$ & .784 \\
\hline$\$ 20,001-\$ 50,000$ & 109 (32.9) & $93(33.3)$ & \\
\hline$\$ 51,001-\$ 75,000$ & $50(15.1)$ & $37(13.3)$ & \\
\hline$\$ 75,001+$ & $62(18.7)$ & $63(22.6)$ & \\
\hline Missing & $40(12.1)$ & $31(11.1)$ & \\
\hline \multicolumn{4}{|l|}{ Marital status } \\
\hline Single & $53(16.0)$ & $64(22.9)$ & .019 \\
\hline Married & $204(61.6)$ & $144(51.6)$ & \\
\hline Separated, divorced, or widowed & $54(16.3)$ & $60(21.5)$ & \\
\hline Missing & $20(6.0)$ & $11(3.9)$ & \\
\hline \multicolumn{4}{|l|}{ Number of people in home } \\
\hline 1 & $15(4.5)$ & $31(11.1)$ & .017 \\
\hline 2 & $71(21.5)$ & $48(17.2)$ & \\
\hline $3-4$ & $98(29.6)$ & $86(30.8)$ & \\
\hline $5+$ & $57(17.2)$ & $36(12.9)$ & \\
\hline Missing & $90(27.2)$ & $78(28.0)$ & \\
\hline
\end{tabular}

in each group. Self-reported eating behaviors scores improved consistently from baseline to 4 months to 10 months in both groups.

Table 4 displays the change over time of the entire population of patients on the primary and secondary outcomes of the study. As a whole, enrolled patients showed significant, albeit small, changes in most areas. The Combined Outcome Score, designed to assess changes in the 3 areas of physical activity, healthy eating, and emotional well-being, showed significant improvement at 10 months for the entire population.

We examined clinically significant changes at the individual patient level. In our direct measurement of BMI and fitness level, $16.2 \%$ of patients who completed a 10 -month visit and $10 \%$ of all patients enrolled lost $5 \%$ or more of their body weight. Similarly, $16.7 \%$ of patients who completed a 10 -month visit and $10.3 \%$ of all patients enrolled had a 2-point or greater increase in their fitness level. One hundred ten patients $(29.2 \%$ of those who completed the 10 -month visit, $18.0 \%$ of all enrollees) had either lost $5 \%$ or more of their body weight and/or increased their fitness level by 2 or more points. Only 14 of the 110 individuals lost more than $5 \%$ of their body weight and increased their fitness levels by 2 points, indicating that most individuals did appear to focus on 1 area for improvement in this time frame. There were no significant differences in these outcomes between the 2 practice groups.

All practices were rated by a composite score intended to assess the fidelity of delivering the intervention: practice efforts to create an internal culture of wellness (scale 6 to 18 ). Site visits indicated that many practices in both groups had existing workplace wellness activities focused on clinician and staff behavioral change independent of the study activities. Several traditional practices independently initiated such activities during the study without direction from the central research study team. Patient-level outcomes were assessed using this fidelity measure as a continuous outcome to determine whether there appeared to be any dose response curves using this measure as a proxy for the intervention dose. Systolic blood pressure was not correlated with this fidelity measure, $P=.486$. All other measures (BMI, PrimeScreen, fitness level, SF-12, and the Combined Outcome Score) were significantly correlated in a positive direction with 
Table 2. Baseline Physiologic Characteristics of Patients in Study Practices

\begin{tabular}{|c|c|c|c|c|c|c|c|}
\hline \multirow[b]{2}{*}{ Variable } & \multicolumn{3}{|c|}{ Enhanced Practice, $\mathbf{n}=331$} & \multicolumn{3}{|c|}{ Traditional Practice, $n=279$} & \multirow{2}{*}{$\begin{array}{l}P \\
\text { Value }^{a}\end{array}$} \\
\hline & Mean & SD & Median & Mean & SD & Median & \\
\hline Continuous descriptors, $t$ test, with median & 43.5 & 11.1 & 44.0 & 44.3 & 13.7 & 45.0 & .425 \\
\hline Systolic blood pressure, mm Hg & 128.5 & 15.1 & 128.0 & 124.4 & 16.5 & 122.0 & .002 \\
\hline Diastolic blood pressure, $\mathrm{mm} \mathrm{Hg}$ & 80.5 & 10.4 & 80.0 & 77.2 & 9.9 & 79.0 & $<.001$ \\
\hline HOMA-IR log transformation & 1.1 & 0.7 & 1.1 & 1.2 & 0.8 & 1.1 & .088 \\
\hline PrimeScreen ${ }^{b}$ AIM-HI & -0.1 & 0.4 & -0.1 & -0.1 & 0.4 & -0.0 & .910 \\
\hline Total MET minutes, square root transformed & 24.9 & 19.7 & 21.3 & 27.6 & 21.2 & 23.0 & .123 \\
\hline \multicolumn{8}{|l|}{ Continuous descriptors, nonparametric test } \\
\hline $\mathrm{BMI}$ & 37.0 & 5.7 & 35.8 & 36.8 & 6.0 & 35.0 & .292 \\
\hline Fitness & 4.1 & 2.0 & 4.0 & 3.7 & 1.9 & 4.0 & .034 \\
\hline SF-12 mentald & -0.3 & 1.1 & -0.2 & -0.3 & 1.1 & -0.1 & .522 \\
\hline \multicolumn{8}{|c|}{$\begin{array}{l}\text { AIM-HI = Americans In Motion-Healthy Interventions; BMI = body mass index. HOMA-IR = homeostatic model assessment: insulin resistance; MET = metabolic } \\
\text { equivalent test; NMRLP = nuclear magnetic resonance lipoprotein profiles; SF-12 = SF-12 Health Survey, a short form subset of the SF-36. }\end{array}$} \\
\hline $\begin{array}{l}\text { a For BMI, fitness, SF- } 12 \text { mental, and SF-12 physical we } \\
\text { PrimeScreen, we used analysis of variance. } \\
\text { b PrimeScreen is a brief dietary screening tool. } \\
\text { 'Scored on a range from } 1 \text { to } 7 \text {, based on heart rate, a } \\
\text { ' }{ }^{d} \text { Mental or physical component summary scores of the }\end{array}$ & nonparamet & Wilcoxon & sum test. FC & iystolic and & olic blooc & essures, HOM & NMRLP, anc \\
\hline
\end{tabular}

this measure, with values ranging from $P<.001$ for the PrimeScreen to $P=.027$ for fitness level.

\section{DISCUSSION}

This study examined patient-level outcomes in primary care practices that attempted to incorporate the AIM-HI approach promoting healthy eating, physical activity, and emotional well-being into routine patient care. This study did not validate the primary hypothesis that the practices which used the AIM-HI tools as a group to improve personal behaviors among clinicians and practice staff, as well as actively advertised their commitment to AIMHI principles to their patients, would be more successful in improving patient-level outcomes. There was a significant difference between practice groups in which patients' systolic blood pressure decreased over time. Blood pressure is responsive to both weight loss and improved physical fitness, making it potentially the most sensitive of the individual measures to the AIM-HI behavioral change approach. The size of the betweengroup difference supports that a decrease in blood pressure is an effect of the intervention, but the higher baseline mean systolic blood pressure in the enhanced practice group could mean that this result represents a regression to the mean instead of an effect of the intervention. The overall negative outcome could be that clinician and practice behavior changes do not translate to patient

\section{Figure 1. Flow diagram of practice selection for study participation and allocation to traditional and enhanced practices.}

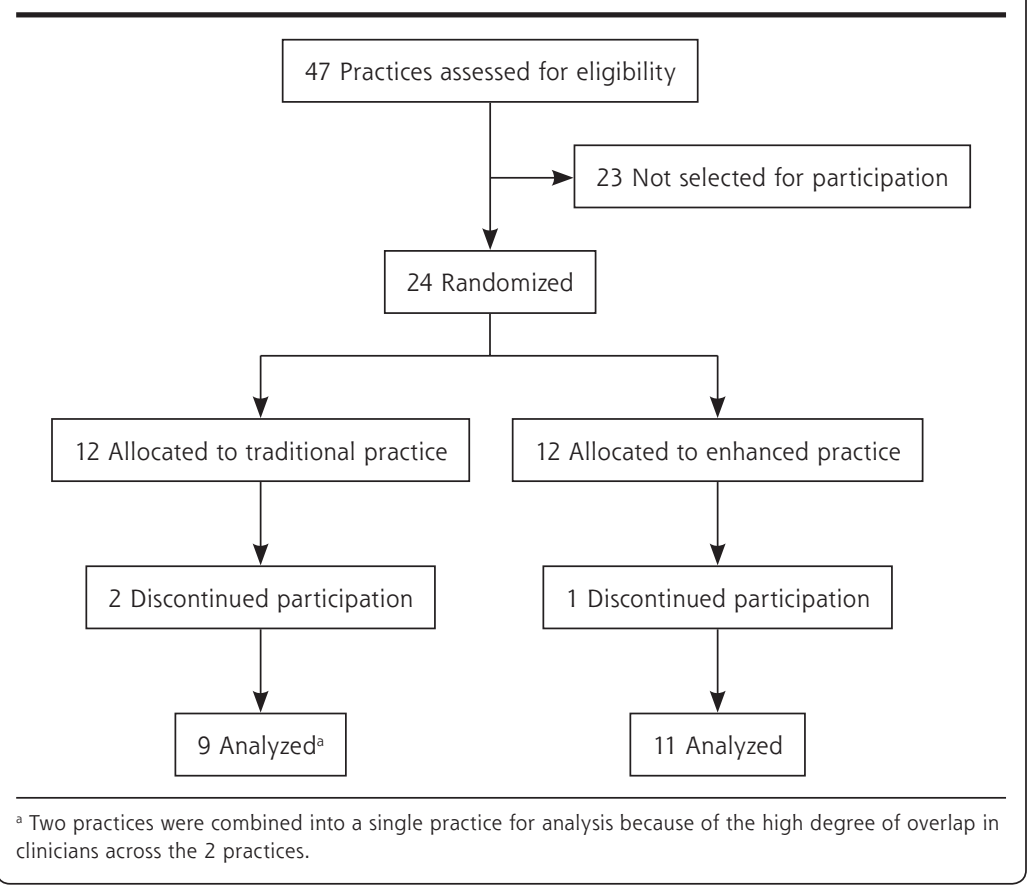


change or that the relatively low intensity of the AIM$\mathrm{HI}$ intervention was inadequate to promote populationlevel physiologic changes. ${ }^{29}$
The AIM-HI approach is designed to be a relatively low-intensity lifestyle-based program that can be incorporated into primary care office visits as brief interactions. The approach is not designed as a high-intensity, directed, behavioral therapy. It is designed to help motivated patients become activated in at least 1 patient-selected area. The combination of a behavioral change of low intensity with relatively infrequent follow-up (as obesity was not a billable diagnosis at the time of the study) likely contributed to the negative results. Furthermore, the outcome measures may not have been sensitive to small, lifestyle-based improvements, but they do represent clinically meaningful outcomes for population level health. The lack of a practice-level effect could also be an artifact of the study recruitment and selection process. Practices interested in behavioral change applied to participate, and a combination of practice location, patient population, and engagement in

Table 3. Patient Outcomes by Randomized Practice Condition: Mixed Effects Multivariate Regression

\begin{tabular}{|c|c|c|c|c|c|c|c|c|c|}
\hline \multirow[b]{2}{*}{$\begin{array}{l}\text { Outcome } \\
\text { by Group }\end{array}$} & \multicolumn{2}{|c|}{ Baseline } & \multicolumn{3}{|c|}{4 Months $^{a}$} & \multicolumn{4}{|c|}{10 Months $^{b}$} \\
\hline & Estimate & SE & Estimate & SE & $\begin{array}{c}\text { Change } \\
\text { From Base } \\
P \text { Value }\end{array}$ & Estimate & SE & $\begin{array}{c}\text { Change } \\
\text { From Base } \\
P \text { Value }\end{array}$ & $\begin{array}{c}\text { Between- } \\
\text { Group } \\
\text { Difference } \\
P \text { Value }\end{array}$ \\
\hline \multicolumn{10}{|l|}{ BMI } \\
\hline Traditional & 37.117 & 0.723 & 36.861 & 0.725 & .031 & 36.917 & 0.726 & .107 & .966 \\
\hline Enhanced & 37.513 & 0.737 & 37.296 & 0.741 & .053 & 37.346 & 0.740 & .133 & \\
\hline \multicolumn{10}{|c|}{ Systolic BP, mm Hg } \\
\hline Traditional & 126.317 & 1.960 & 128.660 & 2.015 & .022 & 126.941 & 2.049 & .552 & .009 \\
\hline Enhanced & 130.391 & 1.939 & 128.652 & 2.020 & .071 & 128.178 & 2.018 & .020 & \\
\hline \multicolumn{10}{|l|}{ Fitnessc } \\
\hline Traditional & 3.705 & 0.341 & 4.027 & 0.346 & .010 & 3.810 & 0.349 & .402 & .406 \\
\hline Enhanced & 4.098 & 0.333 & 4.242 & 0.341 & .212 & 4.256 & 0.340 & .159 & \\
\hline \multicolumn{10}{|c|}{ PrimeScreend AIM-HI } \\
\hline Traditional & -0.027 & 0.042 & 0.068 & 0.043 & .000 & 0.106 & 0.044 & .000 & .061 \\
\hline Enhanced & -0.017 & 0.043 & 0.150 & 0.045 & .000 & 0.168 & 0.045 & .000 & \\
\hline \multicolumn{10}{|l|}{ SF-12 mentale } \\
\hline Traditional & -0.072 & 0.120 & 0.129 & 0.123 & .007 & 0.088 & 0.126 & .038 & .652 \\
\hline Enhanced & -0.112 & 0.121 & 0.091 & 0.128 & .003 & -0.037 & 0.129 & .275 & \\
\hline \multicolumn{10}{|c|}{ EQ-5D health state } \\
\hline Traditional & 65.848 & 2.610 & 65.855 & 2.730 & .997 & 69.309 & 2.806 & .071 & .940 \\
\hline Enhanced & 66.671 & 2.636 & 67.338 & 2.817 & .699 & 70.935 & 2.815 & .013 & \\
\hline \multicolumn{10}{|c|}{ AIM-HI = Americans In Motion-Healthy Interventions; $\mathrm{BMI}=$ body mass index; $\mathrm{BP}=$ blood pressure; $\mathrm{EQ}-5 \mathrm{D}=\mathrm{A}$ visual analog health state score. } \\
\hline 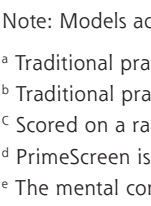 & $\begin{array}{l}\text { eated measur } \\
7 \text { patients; ent } \\
1 \text { patients; en } \\
7 \text {, based on } 1 \\
\text { y screening to } \\
\text { mary of the SP }\end{array}$ & $\begin{array}{l}5, \text { clusterir } \\
\text { anced pra } \\
\text { anced pra } \\
\text { eart rate, } \\
12 \text { Health }\end{array}$ & $\begin{array}{l}\text { patients withi } \\
n=201 \mathrm{pat} \\
\mathrm{n}=206 \mathrm{pa} \\
\text { and sex, with } \\
\text { ey, a short fo }\end{array}$ & $\begin{array}{l}\text { practices, } \\
\text { nts. } \\
\text { nts. } \\
\text { igher scor }\end{array}$ & $\begin{array}{l}\text { indicating grea } \\
\text { the SF-36. }\end{array}$ & Iucation, sex, & come, ma & al status, race, a & d ethnicity. \\
\hline
\end{tabular}


Table 4. Population Level Outcomes Over Time-Mixed Effects Multivariate Regression Model

\begin{tabular}{l}
\cline { 2 - 9 } \\
\cline { 2 - 9 }
\end{tabular}

behavioral change activities was included in the selection criteria for both groups. Thus, both groups of practices were motivated to recommend behavioral change activities to patients before entry into the study.

Although observational studies support the idea that clinician lifestyle behaviors affect counseling activities, ${ }^{30,31}$ these studies do not examine the ability to change clinicians' counseling patterns or effectiveness through personal or practice-level efforts to improve fitness behaviors. This study does not support the hypothesis that interventions aimed to improve physician and office staff lifestyle behaviors translates to improvements in patient outcomes related to lifestyle counseling. This finding could be due to an ineffective patient-level intervention related to the outcomes studied, or it could be due to a lack of effect of practice-based activities on the effectiveness of patientlevel counseling activities.

The study did not meet the original goal of 408 patients completing the 10-month data collection, which means the final power to detect a change was lower than anticipated. Power to detect a moderate, .43, effect size was obtained. With the exception of the self-reported PrimeScreen measure, between-group comparisons at either the combined outcome measure or the individual level did not show trends toward one group or the other, making it unlikely that a larger sample size would have resulted in statistically different outcomes.

Providing intensive behavioral therapy for enrolled patients (for example, weekly visits for a month, twice monthly visit for 5 months, and monthly visits for 6 months) was not attempted in this study, as at the time there was no way to support this level of intervention after the study was over. Thus, the findings of a study that relied on intensive therapy would not have been useful in everyday practice. With the recent decision by the Center for Medicare and Medicaid Services to reimburse primary care practices for intensive behavioral therapy for obesity, implementation studies of more intensive levels of contact in primary care settings would appear warranted.

Though patient-level changes observed across the population were small, the study population overall improved in a number of areas in a before and after comparison. Although this project was conducted in motivated practices that recruited patients with an expressed interest in behavioral change as measured using the transtheoretical framework, it nonetheless suggests that clinicians who address patient lifestyle changes using a nonprescriptive problem-solving format can observe significant behavioral changes in motivated patients. Given the difficulty in establishing and maintaining behavioral change, a primary care practice-based intervention that was associated with $18 \%$ of the study population losing more than $5 \%$ of their body weight or improving their fitness level by 2 or more points may encourage clinicians to address lifestyle issues using nondirective approaches. ${ }^{32}$

Simple nonsmoking messages delivered in primary care have been shown to motivate approximately $5 \%$ of smokers to quit smoking. ${ }^{33,34}$ This level of impact is considered worth the effort involved at the practice level. With obesity and sedentary lifestyles rapidly approaching smoking in their negative impact on the US population health, a similarly brief intervention related to these areas that has a clinically significant impact on more than $18 \%$ of the motivated population may be worthwhile. Studies with longer follow-up will be required to determine whether the changes persist over time. The low-intensity, small changes approach of AIM-HI will not have marked effects on a practice's entire population (nor was the approach intended to do so); therefore, pri- 
mary care clinicians will need to develop other behavioral change options as well, such as hiring embedded behavioral health professionals or creating effective linkages to community-based programs, to broaden the reach of effective behavioral change.

Both practice groups were able to show significant improvements in selected patient-level outcomes with no difference between the 2 groups. This negative finding may indicate that observed effects were entirely based on patient selection, and that individuals in these 2 groups would have made these behavioral changes regardless of whether they participated in the project. Although possible, the percentage of individuals that lost more than $5 \%$ of their body weight is larger than most control groups in primary care weight loss studies. ${ }^{35-37}$ For instance, $5 \%$ of the control group in Martin, et $\mathrm{al}^{36}$ and less than $2 \%$ of the control group in Tsai et $\mathrm{al}^{37}$ lost $5 \%$ or more of their body weight. Furthermore, in Tsai's study the intervention group had also regained their baseline weight by 12 months. Other nonprimary care, intensive, weight loss studies have shown clinically significant weight loss in the range of $20 \%$ of the population for control patients given educational interventions..$^{38,39}$ There is little published information available on changes in fitness levels over time in the obese population; therefore, it is unclear whether the observed changes in fitness levels represent background variation or real improvement at the population level. Thus, the before-after finding may be entirely explained by patient enrollment bias, though finding of this magnitude in control groups have been rare in other primary care-based behavioral change studies.

Other reasons for the lack of practice-level effects are possible. For instance, the nonprescriptive approach to AIM-HI and the tools provided to help begin the conversation may have been reasonably intuitive, and thus easily incorporated into daily practice even without the practice-level activities. The Prescription for Health projects ${ }^{40}$ indicated that practice-level changes of this type were often difficult to implement and sustain; thus, even an intuitive intervention and well-designed tools rarely result in spontaneous changes in practice behavior as shown by patient-level outcomes.

Previous work has found that clinician and practicelevel commitment to healthy lifestyles affects the likelihood of addressing these issues with patients and patient-level outcomes. ${ }^{41}$ Many of the clinicians and practices recruited for the AIM-HI project had already embarked on various forms of workplace wellness activities and were motivated to provide this type of intervention to their patients. Thus, it is possible there was a ceiling effect that the practice-level AIM-HI intervention could not overcome in this group of practices.
The study was designed to assess the impact of the AIM-HI approach in motivated practices and patients, not to assess the ability of the research team to change the level of interest in addressing lifestyle concerns in uninterested practices or clinicians. Patient-level outcomes were correlated with an independent assessment of fidelity of the delivery of the AIM-HI intervention at the practice-level across the 2 groups, supporting the concept that practice commitment to lifestyle behavioral change principles does affect patient outcomes. Though the assessment of how well AIM-HI practices demonstrated healthy activities during a second site visit did not track with study assignment, the changes in practice fitness cultures that were readily apparent in many practices between the 2 site visits may have been related to prestudy characteristics of the practices instead of the AIM-HI practice-level intervention.

Attempts to improve primary care practices' fitness culture using the AIM-HI tools did not translate to differential patient-level improvements in healthy eating, physical activity, or emotional well-being.

To read or post commentaries in response to this article, see it online at http://www.annfammed.org/content/11/4/371.

Key words: health promotion; health behavior; physical activity; obesity; weight loss; practice-based research networks; practice change implementation

Submitted December 5, 2011; submitted, revised, October 12, 2012; accepted November 16, 2012.

Funding support: This research was funded by the Pepsico Foundation, a not-for-profit corporation independent from, though funded by, Pepsico, Inc. After reviewing and approving the grant application, the Pepsico Foundation had no authority over conduct of the project and has not reviewed the content of this manuscript.

\section{References}

1. Fryer GE. Analysis of Medical Expenditure Panel Survey (MEPS), The importance of having a usual source of health care. Am Fam Physician. 2000;62(3):477.

2. American Academy of Family Physicians. Americans In MotionHealthy Interventions (AIM-HI). http://www.aafp.org/online/en/ home/clinical/publichealth/aim.html.

3. American Academy of Family Physicians. AIM-HI Print Materials. Leawood, KS: American Academy of Family Physicians. 2008. Accessed Oct 21, 2009. https://nf.aafp.org/shop/aim-hi.

4. Prochaska JO, Velicer WF. The transtheoretical model of health behavior change. Am J Health Promot. 1997;12(1):38-48.

5. Epstein LH, Wing RR, Koeske R, Ossip D, Beck S. A comparison of lifestyle change and programmed aerobic exercise on weight and fitness changes in obese children. Behav Ther. 1982;13(5):651-665.

6. Rollnick S, Miller WR, Butler C. Motivational Interviewing in Health Care: Helping Patients Change Behavior. New York, NY: Guilford Press; 2008.

7. Hill JO. Understanding and addressing the epidemic of obesity: an energy balance perspective. Endocr Rev. 2006;27(7):750-761. 
8. Hill JO. Can a small-changes approach help address the obesity epidemic? A report of the Joint Task Force of the American Society for Nutrition, Institute of Food Technologists, and International Food Information Council. Am J Clin Nutr. 2009;89(2):477-484.

9. Rodearmel SJ, Wyatt HR, Stroebele N, Smith SM, Ogden LG, Hill JO. Small changes in dietary sugar and physical activity as an approach to preventing excessive weight gain: the America on the Move family study. Pediatrics. 2007;120(4):e869-e879.

10. May ML, Galper LS, Carr JH. Am I Hungry? What to Do When Diets Don't Work. Phoenix, AZ: Nourish Publishing; 2005.

11. Diabetes Prevention Program (DPP) Research Group. The Diabetes Prevention Program (DPP): description of lifestyle intervention. Diabetes Care. 2002;25(12):2165-2171.

12. Ben-Arye E, Lear A, Hermoni D, Margalit RS. Promoting lifestyle self-awareness among the medical team by the use of an integrated teaching approach: a primary care experience. J Altern Complement Med. 2007;13(4):461-469.

13. Novack DH, Suchman AL, Clark W, Epstein RM, Najberg E, Kaplan C. Calibrating the physician. Personal awareness and effective patient care. Working Group on Promoting Physician Personal Awareness, American Academy on Physician and Patient. JAMA. 1997;278(6):502-9.

14. Oberg EB, Frank E. Physicians' health practices strongly influence patient health practices. J R Coll Physicians Edinb. 2009;39(4):290-291.

15. Weyer C, Bogardus C, Mott DM, Pratley RE. The natural history of insulin secretory dysfunction and insulin resistance in the pathogenesis of type 2 diabetes mellitus. J Clin Invest. 1999;104(6):787-794.

16. Cromwell WC, Otvos JD, Keyes MJ, et al. LDL particle number and risk of future cardiovascular disease in the Framingham Offspring Study - implications for LDL management. J Clin Lipidol. 2007;1(6):583-592.

17. El Harchaoui K, van der Steeg WA, Stroes ES, et al. Value of low-density lipoprotein particle number and size as predictors of coronary artery disease in apparently healthy men and women: the EPIC-Norfolk Prospective Population Study. J Am Coll Cardiol. 2007;49(5):547-553.

18. Kraus WE, Houmard JA, Duscha BD, et al. Effects of the amount and intensity of exercise on plasma lipoproteins. $N$ Engl J Med. 2002;347(19):1483-1492.

19. Shadid S, LaForge R, Otvos JD, Jensen MD. Treatment of obesity with diet/exercise versus pioglitazone has distinct effects on lipoprotein particle size. Atherosclerosis. 2006;188(2):370-376.

20. Brodie DA, Inoue A. Motivational interviewing to promote physical activity for people with chronic heart failure. J Adv Nurs. 2005;50(5):518-527.

21. Westman EC, Yancy WS Jr, Olsen MK, Dudley T, Guyton JR. Effect of a low-carbohydrate, ketogenic diet program compared to a low-fat diet on fasting lipoprotein subclasses. Int J Cardiol. 2006;110(2):212-216.

22. Rifas-Shiman SL, Willett WC, Lobb R, Kotch J, Dart C, Gillman MW. Primescreen, a brief dietary screening tool: reproducibility and comparability with both a longer food frequency questionnaire and biomarkers. Public Health Nutr. 2001;4(2):249-254.

23. Craig CL, Marshall AL, Sjostrom M, et al. International physical activity questionnaire: 12-country reliability and validity. Med Sci Sports Exer. 2003;35(8):1381-1395.
24. Allen DD, Wilson M. Introducing multidimensional item response modeling in health behavior and health education research. Health Educ Res. 2006;21(Suppl 1):i73-i84.

25. Brooks R. EuroQol: the current state of play. Health Policy. 1996; 37(1):53-72.

26. Rabin R, de Charro F. EQ-5D: a measure of health status from the EuroQol Group. Ann Med. 2001;33(5):337-343.

27. Ware J Jr, Kosinski M, Keller SD. A 12-Item Short-Form Health Survey: construction of scales and preliminary tests of reliability and validity. Med Care. 1996;34(3):220-233.

28. Golding LA. YMCA Fitness Testing and Assessment Manual. 4th ed. Champaign, IL: Human Kinetics Pub; 2000.

29. U.S. Preventive Services Task Force. Screening for obesity in adults: recommendations and rationale. Ann Intern Med. 2003;139(11): 930-932.

30. Frank E, Rothenberg R, Lewis C, Belodoff BF. Correlates of physicians' prevention-related practices. Findings from the Women Physicians' Health Study. Arch Fam Med. 2000;9(4):359-367.

31. Lobelo F, Duperly J, Frank E. Physical activity habits of doctors and medical students influence their counselling practices. $\mathrm{Br} J$ Sports Med. 2009;43(2):89-92.

32. Lawlor DA, Hanratty B. The effect of physical activity advice given in routine primary care consultations: a systematic review. J Pub Health. 2001;23(3):219-226.

33. Law M, Tang JL. An analysis of the effectiveness of interventions intended to help people stop smoking. Arch Intern Med. 1995;155 (18):1933-1941.

34. Silagy C. Physician advice for smoking cessation. Cochrane Database Syst Rev. 2000;(2):CD000165.

35. Wilson DB, Johnson RE, Jones RM, Krist AH, Woolf SH, Flores SK. Patient weight counseling choices and outcomes following a primary care and community collaborative intervention. Patient Educ Couns. 2010;79(3):338-343.

36. Davis Martin P, Rhode PC, Dutton GR, Redmann SM, Ryan DH, Brantley PJ. A primary care weight management intervention for low-income African-American women. Obesity (Silver Spring). 2006;14(8):1412-1420.

37. Tsai AG, Wadden TA, Rogers MA, Day SC, Moore RH, Islam BJ. A primary care intervention for weight loss: results of a randomized controlled pilot study. Obesity (Silver Spring). 2009;18(8):1614-1618.

38. Appel LJ, Clark JM, Yeh HC, et al. Comparative effectiveness of weight-loss interventions in clinical practice. N Engl J Med. 2011; 365(21):1959-1968

39. Wadden TA, Volger S, Sarwer DB, et al. A two-year randomized trial of obesity treatment in primary care practice. $N$ Engl J Med. 2011;365(21):1969-1979.

40. Annals of Family Medicine. Prescription for Health: changing primary care practice to foster healthy behaviors. 2006. http://www. annfammed.org/content/vol3/suppl_2l.

41. Quintela J, Main DS, Pace WD, Staton EW, Black K. LEAP-A brief intervention to improve activity and diet: a report from CaReNet and HPRN. Ann Fam Med. 2005;3(Suppl 2):S52-S54. 